\title{
COVID 19-Seizing the Host, an Unusual Presentation
}

\author{
Abeera Akram*, Ahmed Nabeel Kazi, and Amritha Alapati \\ Internal Medicine, Saint Mary's Hospital, Waterbury, USA \\ *Corresponding author: Abeera Akram, Internal Medicine, Saint Mary's Hospital, Waterbury, USA, E-mail: abeera.akram@trinityhealthofne.org
}

Received: 06 Apr, 2021 | Accepted: 29 Apr, 2021 | Published: 05 May, 2021

Citation: Akram A, Kazi AN, Alapati A (2021) COVID 19-Seizing the Host, an Unusual Presentation. Clin Res Open Access 7(2): dx.doi.org/10.16966/24696714.166

Copyright: (C) 2021 Akram A, et al. This is an open-access article distributed under the terms of the Creative Commons Attribution License, which permits unrestricted use, distribution, and reproduction in any medium, provided the original author and source are credited.

\section{Introduction}

According to the dashboard by the Center for Systems Science and Engineering at Johns Hopkins University, approximately 95,003,533 cases have been reported up to date. This novel virus has caused $2,029,938$ deaths approximately as of $1 / 17 / 2021$ [1]. This pandemic has not only affected people health-wise but also has a great social, economic, and psychological effect globally.

An investigation report commented on the clinical characteristics of 225 patients with COVID-19. Major clinical symptoms were fever (84.4\%), cough (56.4\%), dyspnea (4\%), expectoration, fatigue, chills, headache, chest pain, and pharyngalgia (3.5 to 22.6\%) [2]. A literature review does show that CNS-associated symptoms can also be a presentation of COVID-19. This is a case report regarding a 49-year-old gentleman who had a unique presentation of COVID-19 infection. He presented with altered mentation and was admitted to the hospital for suspected COVID-19 infection. His hospital course was complicated by an episode of seizure, though he did not have any history of seizures in the past. COVID-19 usually presents with fever, cough, dyspnea, fatigue, etc. We highlight this rare presentation with some literature-reviewed facts in the discussion.

\section{Case Presentation}

A 49-year-old gentleman came into the hospital with nonspecific complaints (which kept on changing) including feeling tired, acid reflux, and cough (which he denied when asked later). He had no significant past medical history. The patient's presentation was not classical for COVID-19 infection considering he denied any shortness of breath, fever, diarrhea, chills, anosmia, or augesia but he was fixated that he has worsening acid reflux which needs to be fixed.

He was an obese, African-American male, alert and oriented to time, place, and person but delusional. Physical examination did not show any neurological deficit. He was hemodynamically stable and initial lab work was significant for hyponatremia $124 \mathrm{meq} / \mathrm{L}$ and no lymphopenia was present on complete blood count. His CT head was negative for any acute hemorrhage or infarct. We spoke to the family to get further history who told us that patient has been at a party a week ago in New Rochelle, he lost 3 of his friends last week because of the COVID-19 infection. Everyone at the party except 2 people was tested positive for COVID-19. Since then the patient has been under stress and delusional thinking that he will die. For high suspicion of COVID-19, his test was sent and he was started on Azithromycin and Plaquenil. On the second day of admission, the rapid response was called because the patient became unresponsive. He was not responding to painful stimuli and his lower lip was shaking. His blood pressure was highly elevated. There was a concern of stroke, stat CT head was repeated which did not show any acute hemorrhage and infarct, and then Electroencephalogram (EEG) showed seizure spikes. His sodium level was 133 meq/L. He was loaded with Levetiracetam and started on Phenytoin. At this point there was a concern that the patient might have encephalitis secondary to COVID-19, his Lumbar puncture was done but it was a dry tap. MRI of the head did not show any encephalitic changes. The patient slowly regained his consciousness. The next day he developed diarrhea, started complaining of cough, and was fixated that he has developed pneumonia and needed treatment. We repeated his chest $\mathrm{x}$-ray which showed no focal lung infiltrate. Unfortunately, he left against medical advice saying that he does not want to get an infection in the hospital. If his test result comes back positive he should be called. A day later, his test came back positive, he was updated and his family brought him to the hospital. According to the family, the day before coming back to the hospital, he passed out three times. During second admission his chest X-ray showed bibasilar infiltrates, D-dimers were also elevated. With the concern of pulmonary embolism as a complication of COVID-19, his CTA chest was done. CTA of the chest showed pulmonary emboli within the right main pulmonary artery as well as segmental and sub-segmental arterial branches of the right upper, right lower, and left upper lobes. It also showed multiple scattered ground-glass densities bilaterally which more pronounced as compared to the prior imaging on his previous admission. In addition to Levonox, he was restarted on Azithromycin, Plaquenil, and anti-epileptics (Levetiracetum and Lacosamide). EEG was repeated which was now negative for seizure. Throughout his hospital stay, he never became hypoxic, his mentation improved, he was closely observed until his inflammatory markers started to trend 
down. Ultimately he was discharged home with instructions to keep himself quarantined for one more week.

\section{Discussion}

COVID-19 infection caused by SARS-CoV-2 novel coronavirus has been a global epidemic of 2020-2021.

Many reports have commented on acute CNS-associated symptoms affected by SARS-CoV-2 infection. Report of 217 hospitalized patients in Wuhan, China, described stroke, encephalopathies, and muscle injuries in patients with severe infection. Anosmia and augesia were also reported. An article also commented on demyelinating and neuromuscular complications which were to occur 2 to 3 weeks after respiratory symptoms but no case presentation with seizures only has been reported [3].

A systemic review regarding the central nervous system manifestations of COVID-19's identified CNS manifestations in $25 \%$ of the patient. Common manifestations were headache, dizziness, impaired consciousness. Seizures were identified in just $0.5 \%$ of the patient but the level of evidence of the study was III. They observed the evidence on the CNS involvement scarce and of low quality [4].

A retrospective study of 214 patients showed $36.4 \%$ had various neurological manifestations that involved the central nervous system, peripheral nervous system, and skeletal muscles. Of patients with severe infection, $5 \%$ had acute cerebrovascular disease, $0.8 \%$ had impaired consciousness and $19.3 \%$ had skeletal muscle injury. Although seizure was included in clinical manifestation of neurological symptoms none was reported [5]. According to a case series published by Lambrecq $\mathrm{V}$, et al. $0.8 \%$ of the COVID-19 positive patients, who were admitted due to severe illness had seizures as their manifesting presentation [6].

A literature review showed the first case of meningitis/encephalitis associated with SARS-CoV-2 which commented on the neuroinvasive potential of the virus. It was regarding a 24-year-old gentleman with fever and transient generalized seizure. Interestingly his nasopharyngeal swab was negative but CSF was positive for SARS-CoV-2 RNA. Unlike our patient, he had MRI findings showing hyperintensity along the wall of the inferior horn of the right lateral ventricle, hyperintense signal change in the right medial temporal lobe and hippocampus, which indicated lateral ventriculitis and encephalitis [7]. Another case report commented on neurological manifestations of a patient with COVID-19 infection. This is a case of a 64-year-old male patient who was tested positive for COVID-19 and 14 days after symptom onset presented with lethargy and unresponsiveness [8].

The potential mechanisms for neuropsychiatric manifestations are thought to be because of viral infiltration into the CNS, cytogenetic dysregulation, and peripheral immune cell transmigration secondary to increased permeability of blood-brain barrier, and post-infectious autoimmunity affecting brain tissue.
Our case report is unique in the way that initially our patient did not present with classic symptoms of COVID-19 infection but he evolved and developed common manifestations including cough, fever, diarrhea as well as pulmonary embolism. His neurological manifestation was an only seizure. It was thought that infection might have caused encephalitis or meningitis but interestingly his MRI did not show any specific changes.

\section{Conclusion}

The burden of this pandemic to the central nervous system is currently unknown but emerging data shows that it is most likely to be significant. In recent outbreaks, symptoms involving CNS are underexplored relative to common presentations. Few patients presented only with neurological manifestations, without classic symptoms (fever, cough, diarrhea, and fatigue) of COVID-19. Therefore physicians are urged to pay close attention to neurological manifestations. Also while seeing a patient with central nervous system involvement we should consider COVID-19 infection in the setting of a global pandemic to avoid misdiagnosis and unintended exposure to care providers. It will help in preventing the transmission of infection to unaffected people.

\section{References}

1. Covid-19 Dashboard at John Hopkins University (2020).

2. Li R, Tian J, Yang F, Lv L, Yu J, et al. (2020) Clinical characteristics of 225 patients with COVID-19 in a tertiary hospital near Wuhan, China. J Clin Virol 127: 104363.

3. Troyer EA, Kohn JN, Hong S (2020) Are we facing a crashing wave of neuropsychiatric sequelae of COVID-19? Neuropsychiatric symptoms and potential immunologic mechanisms. Brain Behav Immun 87: 34-39.

4. Asadi-Pooya, AA, Simani L (2020) Central nervous system manifestations of COVID-19: A systematic review. J Neurol Sci 413: 116832.

5. Mao L, Jin H, Wang M, Hu Y, Chen S, et al. (2020) Neurologic Manifestations of Hospitalized Patients With Coronavirus Disease 2019 in Wuhan, China. JAMA Neurol 77: 683-690.

6. Lambrecq V, Hanin A, Munoz-Musat E, Chougar L, Gassama S, et al. (2021) Association of Clinical, Biological, and Brain Magnetic Resonance Imaging Findings with Electroencephalographic Findings for Patients With COVID-19. JAMA Netw Open 4: e211489.

7. Moriguchi T, Harii N, Goto J, Harada D, Sugawara H, et al. (2020) A first case of meningitis/encephalitis associated with SARSCoronavirus-2. Int J Infect Dis 94: 55-58.

8. Yin R, Feng W, Wang T, Chen G, Wu T, et al. (2020) Concomitant neurological symptoms observed in a patient diagnosed with coronavirus disease 2019. J Med Virol 92: 1782-1784. 